\title{
Adding new dimensions to in situ and operando experiments
}

\author{
Pascal Hofer ${ }^{a}$, Andreas Förster $^{b}$ and Clemens Schulze-Briese ${ }^{c}$ \\ 'DECTRIS USA Inc., 325 Chestnut Street, Suite 800, Philadelphia, PA, USA, pascal.hofer@dectris.com \\ ${ }^{b}$ DECTRIS Ltd., Täfernweg 1, 5405 Baden-Dättwil, Switzerland, andreas.foerster@dectris.com \\ ${ }^{a}$ DECTRIS Ltd., Täfernweg 1, 5405 Baden-Dättwil, Switzerland, clemens.schulze-briese@dectris.com
}

Traditionally, materials involved in energy storage and conversion, magnetism, catalysis, and functional coatings are only examined post-mortem or indirectly, both of which offer limited information regarding the chemical, structural and mechanical evolution under operation. As such, there is a need for in situ non-destructive materials characterization techniques that permit investigation in real time and provide direct insight into cycling processes. In situ and operando powder diffraction setups at synchrotrons take advantage of the high flux and collimation of the X-ray beams. While these measurements provide quantitative phase information, they lack the spatial resolution to understand complex materials during operation. Absorption and phase contrast computed tomography (CT) can fill the gap, but they are of limited value for chemical and structural investigations.

Synchrotron X-ray diffraction tomography (XRD-CT) overcomes these limitations and has proven to be a powerful characterization tool for the nondestructive investigation of functional materials, like heterogeneous catalysts, catalytic membrane reactors and battery cells. With the advent of efficient and fast high-energy detectors, XRD-CT can now be carried out with 5D resolution (3 spatial, 1 scattering and 1 temporal dimension).

Here we describe the method and the experimental setup with special emphasis on detector requirements. We will present highlights from recent research papers to illustrate the power of the XRD-CT at current sources. Future perspectives of XRD-CT enabled by the high brightness available at the upcoming MBA sources in combination with the next generation of high-energy detectors will be discussed. 